\title{
RESPUESTA A ALTOS NIVELES DE ESTRÉS HÍDRICO EN MAÍZ ${ }^{1}$
}

\author{
Carlos Hugo Avendaño-Arrazate ${ }^{2}$, José Domingo Molina-Galan², Carlos Trejo-López $z^{2}$, \\ Cándido López-Castañeda ${ }^{2}$,Jorge Cadena-Iñiguez ${ }^{3}$
}

\begin{abstract}
RESUMEN
Respuesta a altos niveles de estrés hídrico en maíz. El objetivo de este trabajo fue comparar la respuesta a la sequía, en términos de crecimiento vegetativo, desarrollo reproductivo y la acumulación de materia seca, de cuatro variedades de maíz sometidas a diferentes niveles de estrés hídrico, en el Colegio de Postgraduados, México durante el año 2003. Las variedades fueron: Zacatecas 58 original (Zo), Cafime original (Co), Zacatecas 58 SM19 (Z19) y Cafime SM16 (C16). Los niveles de sequía se aplicaron cuando el suelo llegó al punto de marchitez permanente, siendo éstos: 4, 10 y 20 días de sequía, seguidos de la aplicación inmediata de un riego de recuperación y riegos continuos hasta la cosecha. El crecimiento se detuvo por efecto de la sequía en las cuatro variedades, pero una vez que se aplicó el riego de recuperación, éste ocurrió de manera progresiva, de tal forma que las plantas sometidas a sequía igualaron y en algunos casos llegaron a superar a las de riego como ocurrió en Z19. El número de días a floración y asincronía fue mayor en Z19 y C16 que Zo y Co; en el índice de cosecha, las variedades originales disminuyeron menos que las mejoradas; mientras que, la biomasa fue menos afectada en las variedades mejoradas que en las originales conforme se prolongó el periodo de sequía. Por lo anterior, se concluyó que las variedades mejoradas han desarrollado un mecanismo de resistencia a sequía llamado "latencia"; mediante el cual, las plantas detienen su crecimiento en condiciones extremas de sequía, pero en el momento en que nuevamente hay humedad en el suelo, reinician su desarrollo hasta completarlo.
\end{abstract}

\begin{abstract}
Response to high levels of hydric stress in maize. The objective of this work was to compare vegetative and reproductive development and biomass accumulation in four maize varieties under different degrees of water stress in the Postgraduate College, México, 2003. The varieties used were: Zacatecas 58 original (Zo), Cafime original (Co) and, Zacatecas 58 SM19 (Z19), and Cafime SM16 (C16). Drought levels were applied by allowing the soil to reach the permanent wilting point and avoiding Irrigation for 4, 10 and 20 days of drought. This was followed by the immediate application of irrigation to allow plant rehydration, which continued until harvest. Plant growth ceased as a consequence of drought in the four varieties, and resumed after irrigation in such a way that droughted plants equaled and in some cases surpassed irrigated plants, as in Z19. Days to flowering and reproductive asynchrony were higher in Z19 and C16 than in Zo and Co. Total biomass was less affected in the improved than in original varieties. Harvest index fell less in original than in improved varieties with drought severity. The results indicate that varieties improved by mass selection under water stress deployed a "dormancy" resistance mechanism through which plant growth ceases under severe drought, and then resumes when the water is relieved by irrigation or rain.
\end{abstract}

Index words: Zea mays L. drought, asynchrony, days to flowering, drought index.

Palabras clave: Zea mays L., sequía, asincronía, días a floración, índice de sequía.

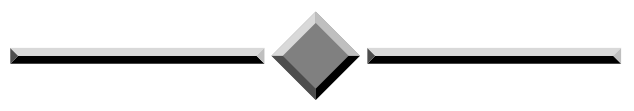

1 Recibido: 9 de mayo, 2007. Aceptado: 18 de enero, 2008. Parte de la Tesis doctoral del primer autor. Posgrado en Recursos Genéticos y Productividad. Colegio de Postgraduados.

2 Colegio de Postgraduados. Carretera México-Texcoco. Km 36,5. CP 56230. México. Autor responsable y para correspondencia: acarlos@ colpos.mx; jomolina@colpos.mx; catre@colpos.mx y lopez@colpos.mx, respectivamente.

3 Colegio de Postgraduados. Campus Salinas, San Luis Potosí. Calle Iturbide No. 73. CP 78600. Salinas de Hidalgo, SLP. México. Correo electrónico: jocadena@colpos.mx 


\section{INTRODUCCIÓN}

En muchas especies de interés antropocéntrico como el maíz (Zea mays L.) se han estudiado las alteraciones que el déficit hídrico provoca en diferentes etapas fenológicas, teniendo un impacto final en los componentes del rendimiento (NeSmith y Ritchie 1992). Estos estudios han podido demostrar que la pérdida en rendimiento de grano es particularmente severa si la sequía ocurre durante la floración o el llenado de grano (Claassen y Shaw 1970, O’Toole y Moya 1981), ya que se ha podido observar que durante la formación de los gametos la planta es altamente sensible al déficit de agua; siendo el gametofito masculino la estructura más sensible, induciendo principalmente esterilidad (Namuco y O'Toole 1986). Los efectos de la sequía en maíz, cuando ocurre en etapas tempranas del desarrollo reproductivo se manifiesta, en una reducción del número de granos, debido principalmente a la absorción del ovario o esterilidad del polen; esto debido a que el déficit hídrico inhibe la fotosíntesis y disminuye el flujo de fotosintatos a los órganos en desarrollo (Boyer y Westgate 2004). Cuando el estrés ocurre durante el desarrollo del cigoto, se provoca aborción o bien el saco embrionario puede ser afectado en su desarrollo pueden ser entre un 15 y $43 \%$ induciendo una asincronía entre la antesis y la floración femenina (Ouattar et al. 1987a, Ober et al. 1991, Desai y Singh 2001). Los bajos potenciales hídricos en maíz provocados por la sequía, contribuyen a detener el crecimiento del embrión, disminuyen el flujo de sacarosa y generan alteración en el metabolismo de los carbohidratos en los ovarios (Zinselmeier et al. 1995). Schussler y Westgate (1991), mencionan que el déficit de agua disminuye la distribución de materia seca, carbohidratos y reduce el nitrógeno en los granos de maíz; sin embargo, la acumulación de carbohidratos en hojas y tallos continúa inmediatamente después de la polinización; por lo que concluyen que la pérdida de grano por sequía es debido en gran parte, a una reducción en el suministro de carbohidratos a la mazorca. Setter et al. (2001) y Schussler y Westgate (1995), mencionan que el flujo de carbohidratos y el ácido abscísico actúan como componentes de un sistema regulatorio por medio del cual la sequía tiene menos efecto en el grano después de la polinización.

Para tener éxito en la formación de la semilla del maíz, según Herrero y Johnson (1981), debe ocurrir una rápida expansión de las estructuras reproductivas, particularmente del pistilo, pero cuando la sequía ocurre durante la antésis, se inhibe el crecimiento del estigma y del jilote, más que el de la espiga; esta diferencia incrementa la asincronía entre la caída del polen y la emergencia del estigma y con ello una falla en la polinización. Moss y Downey (1971), encontraron que la asincronía bajo estrés por sequía, varió entre 13 y 16 días mientras que en plantas que crecieron en condiciones de humedad fue de tres, y la reducción en rendimiento que se ha estimado alcanza valores de entre el 21 y $40 \%$, siendo el peso del grano el componente más afectado (NeSmith y Ritchie 1992).

En México, el mejoramiento genético en maíz para resistencia a sequía se ha enfocado a los componentes de rendimientos (mazorca y grano), siendo uno de los métodos usados para este fin la selección masal visual estratificada (SMVE) (Molina 1980), bajo el supuesto que mediante esta metodología en condiciones de humedad restringida del suelo incrementa la resistencia a sequía. Avendaño et al. (2005), reportan que las variedades Zacatecas 58 y Cafime mejoradas mediante SMVE, han mostrado mayor tolerancia a la sequía y además sugieren que pudieron haber adquirido durante el proceso de mejoramiento el mecanismo de sobrevivencia conocido como "latencia", el cual se caracteriza por que la planta de maíz detiene su crecimiento y entra en un estado de latencia y en el momento que hay nuevamente humedad en el suelo, reinicia su crecimiento hasta completarlo. Con base en lo anterior, se evaluaron cuatro variedades de maíz, procedentes de dos poblaciones obtenidas por SMVE para resistencia a sequía, sometidas a condiciones de humedad restringida, con el objetivo de comparar la respuesta al estrés hídrico en términos de crecimiento vegetativo, desarrollo reproductivo y acumulación de materia seca.

\section{MATERIALES Y MÉTODOS}

\section{Localización}

El trabajo fue realizado en condiciones de invernadero en el Colegio de Postgraduados, México; ubicado ecológicamente en Valles Altos entre $19^{\circ} 29^{\prime} \mathrm{N}$ y $98^{\circ} 53^{\prime} \mathrm{O}$ y $2.250 \mathrm{~m}$ de altitud. El clima es del tipo $\mathrm{Cb}$ (wo)(w)(i')g, correspondiente a templado con verano 
fresco largo, temperatura media anual entre 12 y $18^{\circ}$ C. El mes más frío varía entre 6,5 y $22^{\circ} \mathrm{C}$. La precipitación media anual es de 637 mm (García 1988).

\section{Material biológico}

Se utilizaron las variedades de maíz Zacatecas 58 original (Zo), Cafime original (Co) y sus respectivas variedades mejoradas para resistencia a sequía, Zacatecas 58 SM19 (Z19) y Cafime SM16 (C16), procedentes del ciclo 19 y 16 de selección masal visual estratificada, en un suelo con muy baja humedad (Molina 1980).

\section{Diseño experimental y tratamientos de humedad edáfica}

Se utilizó un diseño experimental de bloques completos al azar con tres repeticiones en un arreglo factorial $4 \times 4$, correspondiendo a tres tratamientos de sequía y uno de riego para cada una de las cuatro poblaciones. Los tratamientos fueron: riego $(\mathrm{R})$ o sequía $(\mathrm{S})$, pudiendo esta última mantenerse por 4, 10, ó 20 días (S1, S2 y S3, respectivamente). Los tratamientos de sequía se iniciaron a partir de que el suelo llegó a punto de marchitez permanente (PMP; S0), es decir, 41 días después de la siembra y el tratamiento de riego consistió en mantener el suelo por arriba del $60 \%$ de humedad aprovechable durante todo el ciclo, equivalente a 140 días. El PMP inició 29 días después del último riego y 41 días acumulados desde la siembra. A todos los tratamientos de sequía se les aplicó un riego de recuperación (RR) una vez alcanzado el periodo determinado de días de sequía a partir del PMP, para continuar con riego normal durante el resto del ciclo. La unidad experimental en cada tratamiento estuvo constituida por dos macetas, cada una con dos plantas haciendo un total de 192 macetas (96 en sequía y 96 en riego).

Se utilizaron como macetas, tubos de PVC de 50 $\mathrm{cm}$ de altura y $10 \mathrm{~cm}$ de diámetro con tapa y perforaciones en el fondo para facilitar el drenaje del exceso de humedad. Cada maceta tuvo 4,25 kg de suelo migajón-arcillo-arenoso que presentó un contenido hídrico a capacidad de campo (CC) de 24,3 \% y PMP de 12,2 $\%$. Las plantas crecieron en el invernadero durante 140 días bajo un régimen de temperatura máxima de $40^{\circ} \mathrm{C}$ y mínima promedio de $9^{\circ} \mathrm{C}$. Cada maceta recibió una fertilización 15 días después de la siembra con la fórmula NPK 40-30-20, usando $27 \mathrm{~g}$ de sulfato de amonio al 20,5\%, $21 \mathrm{~g}$ de superfosfato de Ca simple al $20 \%$ y 4,5 g de cloruro de potasio al $60 \%$. La variación entre efectos de los tratamientos fue determinado por un análisis de varianza de acuerdo con el procedimiento del modelo lineal general del sistema de análisis estadístico y probada la significancia por la prueba de F. La comparación entre medias de variedades y tratamiento de humedad se hizo mediante el método de diferencia mínima significativa (DMS, $\mathrm{p} \leq 0,05$ ).

\section{Variables determinadas}

Se determinaron doce variables durante el ciclo fenológico, de las cuales cuatro estuvieron relacionadas con el crecimiento vegetativo, tres con la reproducción, dos fisiológicas y tres con el rendimiento.

\section{Variables vegetativas}

Altura de planta (AP, cm), se registró midiendo la longitud total desde la base del tallo hasta la base de la espiga.

Aparición y número de hojas (AH, número), se cuantificó cada tercer día, a partir de la aparición de la primera hoja verdadera.

Diámetro basal del tallo (DBT) se midió en cm en la base del tallo al final del ciclo.

Área foliar de la hoja de la mazorca (AFHM, $\mathbf{c m}^{2}$ ) se estimó mediante la ecuación: LA x AH x 0,75; donde $\mathrm{LH}=$ largo de la hoja $\mathrm{AH}=$ ancho de la hoja.

\section{Variables reproductivas}

Días a floración masculina (DFM), se calculó, considerando la fecha en que el $100 \%$ de las espigas mostraron anteras en dehiscencia en cada unidad experimental.

Días a floración femenina (DFF), se calculó, considerando la fecha en que el $100 \%$ de las plantas 
mostraron jilotes con estigmas expuestos en cada unidad experimental.

Asincronía floral en número de días (ASI), se calculó haciendo la diferencia entre DFF y DFM, usando la ecuación: ASI=DFF-DFM.

\section{Variables del rendimiento}

Biomasa total (BT, g), se determinó, pesando peso seco de las plantas cosechadas en cada unidad experimental.

Relación raíz-vástago (RRV), se estimó dividiendo el peso seco de la raíz y el peso seco del vástago, mediante la ecuación: RRV= PSR/PSV; donde: PSR=Peso seco de raíz y PSV=Peso seco del vástago.

Porcentaje de disminución de la biomasa (PDBM, \%), se calculó con la siguiente ecuación: PDBM \%=[1-(BMS/BMR) ${ }^{* 100}$; donde: BMS= Biomasa en sequía y $\mathrm{BMR}=$ Biomasa en riego.

\section{Variables fisiológicas}

Intensidad de sequía(IS), se calculócon la siguiente ecuación: IS=[(RENRi-RENSi)/(RENRi)]/[(RENRRENS)/(RENR)]; donde: RENSi=Rendimiento en sequía de la variedad i; RENRi=Rendimiento en riego de la variedad $\mathrm{i}$; RENR=Rendimiento promedio de todas los variedades en riego y RENS=Rendimiento promedio de las cuatro variedades bajo sequía.
Índice de cosecha (IC), se estimó dividiendo el peso seco del grano (rendimiento económico) entre el peso seco del tallo (rendimiento biológico), mediante la ecuación: $\mathrm{IC}=(\mathrm{RE} / \mathrm{RB}) * 100$; donde: $\mathrm{RE}=\mathrm{Rendimiento}$ económico y $\mathrm{RB}=$ Rendimiento biológico.

\section{RESULTADOS Y DISCUSIÓN}

El análisis de varianza realizado a diez de las variables evaluadas, mostró, que con excepción del porcentaje de disminución de biomasa (PDBM) e índice de sequía (IS) para el caso de variedades, así como, éstas, más la asincronía (ASI) para el caso de tratamientos, todas las demás variables fueron estadísticamente diferentes (Cuadro 1). Con relación a la interacción variedades por tratamientos, ninguna variable mostró diferencias significativas.

\section{Variables vegetativas}

Con relación al crecimiento de planta, la tasa se redujo notablemente ocho días antes de que el suelo llegara a PMP (34 días acumulados desde siembra) y durante todo el periodo de sequía (Figura 1). Lo anterior pudo atribuirse a la restricción de humedad por aplicación de tratamientos, sin embargo, una vez que se aplicó el riego de recuperación $(\mathrm{RR})$, la tasa de crecimiento se reanudó e incrementó en forma exponencial, ocurriendo de forma diferida entre tratamientos. El inicio de la recuperación del crecimiento después de que se aplicó el RR ocurrió

Cuadro 1. Cuadrados medios del análisis de varianza para las variables reproductivas, fisiológicas y de rendimiento de cuatro variedades de maíz y cuatro condiciones de humedad, en invernadero. Colegio de Postgraduados, México. 2003.

\begin{tabular}{lcllcccccccc}
\hline \multicolumn{1}{c}{ F.V. } & G.L. & \multicolumn{1}{c}{ DFM } & \multicolumn{1}{c}{ DFF } & ASI & BMT & RRV & IC & AFHM & DBT & PDBM & IS \\
\hline Var & 3 & $1.107,9^{* *}$ & $1.539,4^{* *}$ & $36,0^{* *}$ & $1.776,5^{* *}$ & $0,07^{* *}$ & $2211,3^{* *}$ & $30.915,4^{* *}$ & $14,7 * *$ & $25,3 \mathrm{~ns}$ & $0,02 \mathrm{~ns}$ \\
Tra & 3 & $803,4^{* *}$ & $754,7^{* *}$ & $20,1 \mathrm{~ns}$ & $2.939,3^{* *}$ & $0,05^{* *}$ & $1793,4^{* *}$ & $26.924,2^{* *}$ & $12,1^{* *}$ & $3.062 \mathrm{~ns}$ & $0,01 \mathrm{~ns}$ \\
Var*Tra & 9 & $11,4 \mathrm{~ns}$ & $11,8 \mathrm{~ns}$ & $13,7 \mathrm{~ns}$ & $59,0 \mathrm{~ns}$ & $0,01 \mathrm{~ns}$ & $102,5 \mathrm{~ns}$ & $624,7 \mathrm{~ns}$ & $0,4 \mathrm{~ns}$ & $12,0 \mathrm{~ns}$ & $0,01 \mathrm{~ns}$ \\
Error & 32 & 7,9 & 15,5 & 7,6 & 91,4 & 0,01 & 0,01 & 221,2 & 0,82 & 0,82 & 0,14 \\
\hline
\end{tabular}

FV=Fuente de variación, Var=variedades, Tra=tratamiento de humedad; Var*Tra=interacción variedades por tramiento, NS=no significativo con un $\alpha=0,05 ; *=$ significativo con $\alpha=0,05$ y **=altamente significativo con $\alpha=0.01$; DFM=días a floración masculina, $\mathrm{DFF}=$ días a floración femenina, ASI=asincronía (días), BMT=biomasa total (g), RRV=relación raíz vástago, IC=índice de cosecha, IS=índice de susceptibilidad a la sequía, DBT=diámetro basal del tallo $(\mathrm{cm})$, PDBM=porcentaje de disminución de la biomasa (\%), AFHM=área foliar de la hoja de la mazorca $\left(\mathrm{cm}^{2}\right)$. 


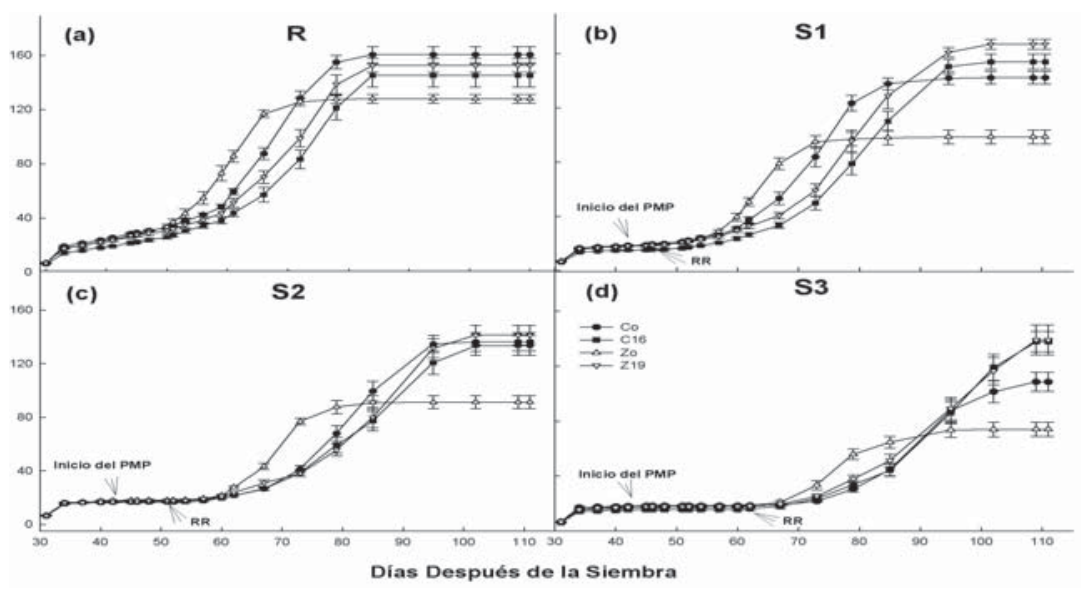

Figura 1. Crecimiento en altura de planta de cuatro variedades de maíz, bajo riego y tres niveles de sequía (S1, S2, S3) a 4, 10 y 20 días después de la condición de punto de marchitez permanente (PMP) respectivamente. Cada punto es el promedio de tres repeticiones \pm error estándar. Colegio de Postgraduados, México. 2003.

a los 14, 10 y 6 días para S1, S2 y S3, respectivamente, de tal forma que la recuperación en las plantas bajo S1 igualaron en altura a las de riego, como fue observado en Z19 bajo sequía, cuyas plantas rebasaron a Z19 bajo riego (Figura 1a). De igual forma, fue observado que en los tratamientos S2 y S3, algunas plantas igualaron en altura a las de riego (Figura $1 \mathrm{a}, \mathrm{c}$ ).
Respecto a la variable de aparición de hojas $(\mathrm{AH})$, se registró un patrón similar al observado en altura de planta, de tal forma que en promedio, los tratamientos de sequía inhibieron la aparición de hojas, cesando ésta en la séptima hoja. La duración de esta inhibición fue atribuible a la restricción de la humedad, ya que una vez que se aplicó el riego de

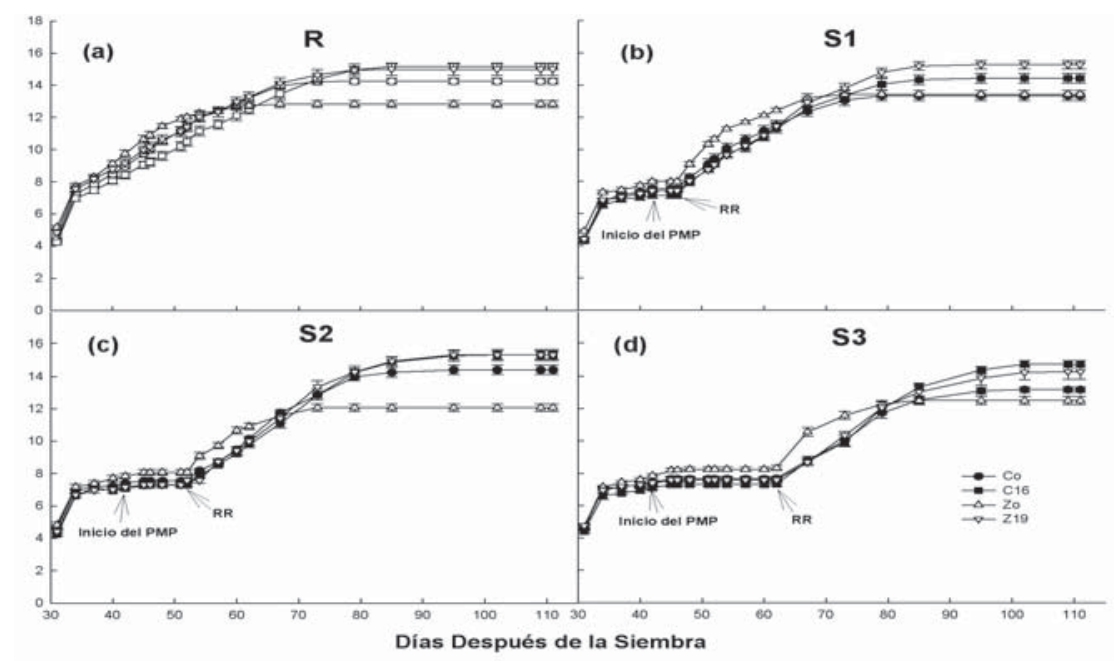

Figura 2. Aparición de hojas en cuatro variedades de maíz, bajo riego y tres niveles de sequía (S1, S2, S3) a 4, 10 y 20 días después de la condición de punto de marchitez permanente (PMP) respectivamente. Cada punto es el promedio de tres repeticiones \pm error estándar. Colegio de Postgraduados, México. 2003. 
recuperación, la aparición de hoja fue normalizada (Figura 2 a, c). Con relación al área foliar de la hoja de la mazorca (AFHM), y diámetro basal del tallo (DBT), su crecimiento estuvo seriamente afectado por la falta de humedad, ya que conforme aumentó la intensidad de la sequía; los valores de ambas disminuyeron gradualmente, aunque la disminución del AFHM fue mayor en las variedades originales que en las mejoradas; sin embargo, para DBT ocurrió lo contrario (Cuadro 2 y 3). Diferentes autores coinciden en que la sequía afecta en general el crecimiento y desarrollo de las plantas (Outtar et al. 1987b, NeSmith y Ritchie 1992), y han relacionado estrechamente la reducción del área foliar con reducción del rendimiento (Saini y
Westgate 2000), estas afirmaciones son confirmados con los resultados obtenidos en esta investigación.

\section{Variables reproductivas}

Los resultados del presente estudio indicaron que el proceso reproductivo fue afectado por la restricción de humedad. Conforme se incrementó la intensidad de la sequía, el número de DFM y DFF se incrementó en las cuatro variedades mostrando diferencias significativas (Cuadro 2). En el caso de la variedad Co, al pasar de riego $(\mathrm{R})$ a $\mathrm{S} 3$, el retraso en número de DFM fue de 23 días (Cuadro 2), mientras que para C16 fue de 16,5;

Cuadro 2. Comparación de medias de variedades de maíz, de las variables reproductivas, fisiológicas y de rendimiento en riego y sequía en invernadero. Colegio de Postgraduados, México. 2003.

\begin{tabular}{|c|c|c|c|c|c|c|c|c|c|c|}
\hline & DFM & DFF & ASI & BMT & RRV & IC & AFHM & DBT & PDBM & IS \\
\hline \multicolumn{11}{|l|}{ Co } \\
\hline $\mathrm{R}$ & $69,1 \mathrm{c}$ & $82,1 \quad \mathrm{c}$ & $12,9 \mathrm{a}$ & 53,6 a & $0,21 \mathrm{ab}$ & $41,1 \mathrm{a}$ & $250,1 \mathrm{a}$ & $10,7 \mathrm{a}$ & & \\
\hline $\mathrm{S} 1$ & $73,9 \mathrm{c}$ & $83,0 \mathrm{c}$ & $9,1 \quad \mathrm{a}$ & $39,3 \mathrm{ab}$ & $0,10 \mathrm{~b}$ & $49,2 \mathrm{a}$ & $146,4 \mathrm{~b}$ & $9,3 \mathrm{ab}$ & $26,7 \mathrm{a}$ & $0,97 \mathrm{a}$ \\
\hline S2 & $81,7 \mathrm{~b}$ & $91,8 \mathrm{~b}$ & $10,1 \mathrm{a}$ & $29,6 \mathrm{~b}$ & $0,19 \mathrm{ab}$ & $33,2 \mathrm{a}$ & $144,1 \mathrm{~b}$ & $9,1 \mathrm{ab}$ & $46,1 \mathrm{a}$ & $0,95 \mathrm{a}$ \\
\hline S3 & $92,1 \mathrm{a}$ & $100,3 \mathrm{a}$ & 8,1 a & $23,3 \mathrm{~b}$ & $0,32 \mathrm{a}$ & $17,3 \mathrm{a}$ & $104,9 \mathrm{~b}$ & $8,2 \mathrm{~b}$ & $56,1 \mathrm{a}$ & $0,94 \mathrm{a}$ \\
\hline DMS & 6,5 & 7,8 & 7,8 & 17,8 & 0,16 & 45,9 & 44,3 & 2,1 & 39,3 & 0,62 \\
\hline \multicolumn{11}{|l|}{ C16 } \\
\hline $\mathrm{R}$ & $80,0 \mathrm{c}$ & $95,0 \quad b$ & $15,0 \mathrm{a}$ & $76,5 \mathrm{a}$ & $0,39 \mathrm{a}$ & $30,4 \mathrm{a}$ & 279,2 a & $12,6 \mathrm{a}$ & & \\
\hline $\mathrm{S} 1$ & 84,4 bc & $94,4 \quad b$ & $10,0 \mathrm{a}$ & $54,1 \mathrm{ab}$ & $0,17 \mathrm{c}$ & $42,7 \mathrm{a}$ & $223,3 \mathrm{ab}$ & $10,7 \mathrm{~b}$ & $29,0 \mathrm{a}$ & $1,04 \mathrm{a}$ \\
\hline S2 & $91,3 \mathrm{ab}$ & $102,8 \mathrm{ab}$ & $11,5 \mathrm{a}$ & $40,1 \mathrm{~b}$ & $0,21 \mathrm{c}$ & $18,1 \mathrm{a}$ & $178,1 \mathrm{ab}$ & $10,4 \mathrm{~b}$ & $47,9 \mathrm{a}$ & $1,12 \mathrm{a}$ \\
\hline S3 & 96,5 a & 108,6 a & $12,1 \mathrm{a}$ & $30,5 \mathrm{~b}$ & $0,28 \mathrm{~b}$ & 9,3 a & $158,5 \mathrm{~b}$ & $10,0 \mathrm{~b}$ & $59,1 \mathrm{a}$ & $1,02 \mathrm{a}$ \\
\hline DMS & 9,0 & 12,4 & 5,5 & 32,9 & 0,06 & 35,9 & 101,1 & 1,5 & 47,5 & 0,88 \\
\hline \multicolumn{11}{|l|}{ Zo } \\
\hline $\mathrm{R}$ & $60,1 \mathrm{~b}$ & $68,1 \mathrm{c}$ & 7,9 a & $39,0 \mathrm{a}$ & $0,14 \mathrm{a}$ & 57,9 a & $158,2 \mathrm{a}$ & $9,9 \mathrm{a}$ & & \\
\hline S1 & $64,8 \mathrm{~b}$ & $71,3 \mathrm{bc}$ & 6,5 a & $26,5 \mathrm{ab}$ & $0,05 \mathrm{~b}$ & $62,0 \mathrm{a}$ & $100,5 \mathrm{~b}$ & 8,6 a & $26,0 \mathrm{a}$ & $0,97 \mathrm{a}$ \\
\hline S2 & $70,2 \mathrm{~b}$ & $77,1 \mathrm{~b}$ & 6,9 a & $19,6 a b$ & $0,05 \mathrm{~b}$ & $63,7 \mathrm{a}$ & 87,0 bc & $7,7 \mathrm{a}$ & $43,1 \mathrm{a}$ & $1,12 \mathrm{a}$ \\
\hline S3 & $74,2 \mathrm{a}$ & $87,1 \mathrm{a}$ & $12,5 \mathrm{a}$ & $14,0 \mathrm{~b}$ & $0,08 \mathrm{ab}$ & $37,7 \mathrm{a}$ & $70,5 \mathrm{c}$ & $8,5 \mathrm{a}$ & $61,1 \mathrm{a}$ & $0,95 \mathrm{a}$ \\
\hline DMS & 5,0 & 7,3 & 7,2 & 23,7 & 0,07 & 42,5 & 20,6 & 2,6 & 60,2 & 0,7 \\
\hline \multicolumn{11}{|l|}{ Z19 } \\
\hline $\mathrm{R}$ & $77,7 \mathrm{c}$ & 90,9 b & $13,1 \mathrm{a}$ & $73,6 \mathrm{a}$ & $0,35 \mathrm{a}$ & $30,3 \mathrm{a}$ & $265,6 \mathrm{a}$ & $12,5 \mathrm{a}$ & & \\
\hline S1 & $83,7 \mathrm{bc}$ & $97,4 \mathrm{~b}$ & $13,6 \mathrm{a}$ & $54,8 a b$ & $0,15 \mathrm{a}$ & $34,7 \mathrm{a}$ & $206,3 \mathrm{a}$ & $10,2 \mathrm{a}$ & $23,3 \mathrm{a}$ & $1,07 \mathrm{a}$ \\
\hline S2 & $88,6 \mathrm{~b}$ & $97,8 \mathrm{~b}$ & $9,1 \quad \mathrm{a}$ & $38,8 a b$ & $0,20 \mathrm{a}$ & $38,2 \mathrm{a}$ & $194,1 \mathrm{a}$ & $10,1 \mathrm{a}$ & $44,8 \mathrm{a}$ & $1,04 \mathrm{a}$ \\
\hline S3 & $99,5 \mathrm{a}$ & $111,3 \mathrm{a}$ & $11,8 \mathrm{a}$ & $31,5 \mathrm{~b}$ & $0,21 \mathrm{a}$ & $10,0 \mathrm{a}$ & $181,1 \mathrm{a}$ & $10,3 \mathrm{a}$ & 55,6 a & $1,13 \mathrm{a}$ \\
\hline DMS & 8,1 & 12,4 & 7,9 & 38,4 & 0,2 & 46,6 & 111,7 & 2,8 & 41,1 & 0,86 \\
\hline
\end{tabular}

Medias con la misma letra (s) entre tratamientos son estadísticamente iguales con un $\alpha=0,05$.

$\mathrm{R}=$ riego, y tres niveles de sequía (S1, S2, S3) a 4, 10 y 20 días después de la condición de punto de marchitez permanente (PMP) respectivamente.

DMS=diferencia mínima significativa, DFM=días a floración masculina, DFF=días a floración femenina, ASI=asincronía (días), $\mathrm{BMT}=$ biomasa total $(\mathrm{g}), \mathrm{RRV}=$ relación raíz vástago, IC=índice de cosecha, AFHM=área foliar de la hoja de la mazorca $\left(\mathrm{cm}^{2}\right)$, DBT=diámetro basal del tallo (cm), PDBM=porcentaje de disminución de la biomasa (\%), IS=índice de susceptibilidad a la sequía. 
Cuadro 3. Comparación de medias de los tratamientos de riego y sequía de la variables reproductivas, fisiológicas y de rendimiento en cuatro variedades de maíz en invernadero. Colegio de Postgraduados, México. 2003.

\begin{tabular}{|c|c|c|c|c|c|c|c|c|c|c|}
\hline & DFM & DFF & ASI & BMT & RRV & IC & AFHM & DBT & PDBM & IS \\
\hline \multicolumn{11}{|l|}{$\mathbf{R}$} \\
\hline Co & $69,1 \mathrm{~b}$ & $82,1 \mathrm{~b}$ & $12,9 \mathrm{a}$ & $53,6 \mathrm{ab}$ & $0,21 \mathrm{ab}$ & $41,1 \mathrm{ab}$ & $250,1 \mathrm{a}$ & $10,7 \mathrm{ab}$ & & \\
\hline $\mathrm{C} 16$ & 80,0 a & $95,0 \mathrm{a}$ & $15,0 \mathrm{a}$ & $76,5 \mathrm{a}$ & $0,39 \mathrm{a}$ & $30,4 \mathrm{~b}$ & $279,2 \mathrm{a}$ & $12,6 \mathrm{a}$ & & \\
\hline Zo & $60,1 \mathrm{c}$ & $68,1 \mathrm{c}$ & $7,9 \quad b$ & $39,0 \mathrm{~b}$ & $0,14 \mathrm{~b}$ & 57,9 a & $158,5 \mathrm{~b}$ & $9,9 \quad \mathrm{~b}$ & & \\
\hline Z19 & 77,7 a & 90,9 a & $13,1 \mathrm{a}$ & $73,6 \mathrm{a}$ & $0,35 \mathrm{ab}$ & $30,3 \mathrm{~b}$ & $265,6 \mathrm{a}$ & $12,5 \mathrm{a}$ & & \\
\hline DMS & 5,5 & 6,7 & 4,6 & 24,1 & 0,21 & 21,4 & 79,8 & 2,0 & & \\
\hline \multicolumn{11}{|l|}{ S1 } \\
\hline Co & $73,9 \mathrm{~b}$ & $83,0 \mathrm{bc}$ & $9,1 \quad \mathrm{a}$ & $39,3 \mathrm{ab}$ & $0,10 \mathrm{ab}$ & $49,2 \mathrm{ab}$ & $146,4 \mathrm{~b}$ & $9,3 \mathrm{ab}$ & $26,7 \mathrm{a}$ & $0,97 \mathrm{a}$ \\
\hline $\mathrm{C} 16$ & $84,4 \mathrm{a}$ & $94,4 \mathrm{ab}$ & $10,0 \mathrm{a}$ & $54,1 \mathrm{a}$ & $0,17 \mathrm{a}$ & $42,7 \mathrm{ab}$ & $223,3 \mathrm{a}$ & $10,7 \mathrm{a}$ & $29,0 \mathrm{a}$ & $1,02 \mathrm{a}$ \\
\hline Zo & $64,8 \mathrm{c}$ & $71,3 \mathrm{c}$ & $6,5 \mathrm{a}$ & $26,5 \mathrm{~b}$ & $0,05 \mathrm{~b}$ & $62,0 \mathrm{a}$ & $100,5 \mathrm{c}$ & 8,6 b & $26,0 \mathrm{a}$ & $0,97 \mathrm{a}$ \\
\hline Z19 & 83,7 a & 97,4 a & $13,6 \mathrm{a}$ & 54,8 a & $0,15 \mathrm{a}$ & $34,7 \mathrm{~b}$ & $206,3 \mathrm{a}$ & $10,2 \mathrm{ab}$ & 23,3 a & $1,07 \mathrm{a}$ \\
\hline DMS & 7,2 & 11,7 & 8,3 & 16,7 & 0,09 & 26,8 & 38,1 & 1,9 & 24,5 & 0,26 \\
\hline \multicolumn{11}{|l|}{ S2 } \\
\hline Co & $81,7 \mathrm{~b}$ & $91,8 \quad b$ & $10,1 \mathrm{a}$ & $29,6 \mathrm{a}$ & $0,19 \mathrm{a}$ & $33,2 \mathrm{ab}$ & $144,1 \mathrm{ab}$ & $9,1 \quad a b$ & $46,0 \mathrm{a}$ & $0,95 \mathrm{a}$ \\
\hline $\mathrm{C} 16$ & $91,3 \mathrm{a}$ & $102,8 \mathrm{a}$ & $11,5 \mathrm{a}$ & $40,1 \mathrm{a}$ & $0,21 \mathrm{ab}$ & $18,1 \mathrm{~b}$ & $178,1 \mathrm{a}$ & $10,4 \mathrm{a}$ & $47,9 \mathrm{a}$ & $1,02 \mathrm{a}$ \\
\hline Zo & $70,2 \mathrm{c}$ & $77,1 \quad \mathrm{c}$ & 6,9 a & $19,6 \mathrm{a}$ & $0,05 \mathrm{~b}$ & $63,7 \mathrm{a}$ & $87,0 \quad b$ & $7,7 \quad b$ & $43,1 \mathrm{a}$ & $1,12 \mathrm{a}$ \\
\hline Z19 & $88,6 \mathrm{ab}$ & $97,8 \mathrm{ab}$ & $9,1 \quad$ a & $38,8 \mathrm{a}$ & $0,20 \mathrm{a}$ & $38,2 \mathrm{ab}$ & $194,1 \mathrm{a}$ & $10,2 \mathrm{ab}$ & $44,8 \mathrm{a}$ & $1,04 \mathrm{a}$ \\
\hline DMS & 8,8 & 9,5 & 5,7 & 29,9 & 0,14 & 37,1 & 79,1 & 2,7 & 27,8 & 0,94 \\
\hline \multicolumn{11}{|l|}{ S3 } \\
\hline Co & $92,1 \mathrm{a}$ & 100,3 a & 8,1 a & 23,3 a & $0,32 \mathrm{a}$ & $17,3 \mathrm{a}$ & $104,9 a b$ & 8,2 a & 56,0 a & $0,94 \mathrm{a}$ \\
\hline $\mathrm{C} 16$ & $96,5 \mathrm{a}$ & 108,6 a & $12,1 \mathrm{a}$ & $30,5 \mathrm{a}$ & $0,28 \mathrm{a}$ & 9,3 a & $158,5 \mathrm{ab}$ & $10,0 \mathrm{a}$ & $59,1 \mathrm{a}$ & $1,04 \mathrm{a}$ \\
\hline Zo & $74,5 \mathrm{~b}$ & $87,1 \quad b$ & $12,6 \mathrm{a}$ & $14,0 \mathrm{a}$ & $0,08 \mathrm{~b}$ & $37,7 \mathrm{a}$ & $70,5 \mathrm{~b}$ & 8,5 a & $61,1 \mathrm{a}$ & $0,95 \mathrm{a}$ \\
\hline Z19 & 99,5 a & $111,3 \mathrm{a}$ & $11,8 \mathrm{a}$ & $31,5 \mathrm{a}$ & $0,21 \mathrm{a}$ & $10,0 \mathrm{a}$ & $181,1 \mathrm{a}$ & $10,3 \mathrm{a}$ & 55,6 a & $1,13 \mathrm{a}$ \\
\hline DMS & 7,4 & 12,1 & 9,1 & 33,0 & 0,12 & 45,3 & 105,5 & 2,7 & 42,6 & 0,99 \\
\hline
\end{tabular}

Medias con la misma letra (s) entre tratamientos son estadísticamente iguales con un $\alpha=0,05$.

$\mathrm{R}=$ riego, y tres niveles de sequía (S1, S2, S3) a 4, 10 y 20 días después de la condición de punto de marchitez permanente (PMP) respectivamente.

DMS=diferencia mínima significativa, DFM=días a floración masculina, DFF=días a floración femenina, ASI=asincronía (días), $\mathrm{BMT}=$ biomasa total $(\mathrm{g}), \mathrm{RRV}=$ relación raíz vástago, IC=índice de cosecha, $\mathrm{AFHM=área} \mathrm{foliar} \mathrm{de} \mathrm{la} \mathrm{hoja} \mathrm{de} \mathrm{la} \mathrm{mazorca}\left(\mathrm{cm}^{2}\right)$, DBT=diámetro basal del tallo (cm), PDBM=porcentaje de disminución de la biomasa (\%), IS=índice de susceptibilidad a la sequía.

14,4 para Zo y 21,8 en Z19 (Cuadro 3). Referente a la floración femenina, el retraso en número de DFF fue de 18,2 en Co y 13,6 en C16; mientras que en Zo fue de 19 y 20,4 en Z19. En el caso de Cafime, la variedad mejorada presentó un menor retraso en las floraciones, mientras que en Zacatecas 58 sucedió lo contrario. El retraso en ambas floraciones por falta de humedad pudo haber provocado daños en la viabilidad del polen, como fue observado por Schoper et al. (1986), y en la receptividad del estigma como lo señalan Basseti y 
Westgate (1993), y consecuentemente en el proceso de polinización como lo señalan Ekanayake et al. (1990), y Sawhney y Shukla (1994).

Generalmente se ha reportado que existe menor asincronía entre la floración masculina y femenina en genotipos de maíz cuando el desarrollo ocurre en condiciones de humedad (O’Toole y Namuco 1983). Sin embargo, en el presente estudio, los valores de asincronía tendieron a ser mayores en riego que en sequía (Cuadro 2), donde las variedades mejoradas mostraron mayor asincronía floral que las originales. La excepción ocurrió en S3 ya que la asincronía de Zo resultó ligeramente mayor que la de Z19. Una interpretación a estos resultados sería que la planta disminuye la asincronía floral por efecto del estrés hídrico como un mecanismo de sobrevivencia.

\section{Variables de rendimiento}

En las cuatro variedades, la biomasa total (BMT) se redujo gradualmente con el aumento de la intensidad de la sequía (Cuadro 2 y 3). La BMT fue mayor en las variedades mejoradas que en las originales y se mantuvo en los tres tratamientos de sequía. Este mismo comportamiento lo tuvo el porcentaje de disminución de la biomasa (PDBM).

En la comparación entre variedades dentro de cada tratamiento, aún cuando no se observaron diferencias significativas, Co presentó menor PDBM que C16, mientras que en Zacatecas 58 ocurrió lo contrario, ya que Zo fue mayor en PDBM que Z19; este comportamiento se pudo deber a que las variedades mejoradas después del RR tuvieron una tasa de crecimiento (altura y número de hojas) similar a las plantas que estuvieron en riego continuo, especialmente Z19. El aumento en altura y número de hojas trajo consigo un incremento de la biomasa, debido probablemente a que estas variedades han desarrollado durante el proceso de selección, diferentes mecanismos de sobrevivencia, como el mencionado por Muñoz et al. (1983), Graff (1989), y Avendaño et al. (2005), denominado latencia, mediante el cual las plantas detienen su crecimiento en condiciones extremas de sequía, y en el momento en que hay nuevamente humedad en el suelo, reinician su desarrollo hasta completarlo como lo reportan Neale et al. (2000) en el pasto Sporobolus stapfianus en condiciones severas de sequía. De esta forma, es posible que mediante este mecanismo las variedades mejoradas tengan la capacidad de almacenar fotosintatos aún en bajos potenciales hídricos de la planta. Al respecto, Bradford (1994) menciona que las relaciones hídricas en el desarrollo de las plantas son determinantes para resistir el déficit hídrico, debido a que el transporte continuo del floema requiere de bajos potenciales hídricos en las regiones de demanda para atraer fotosintatos y agua.

\section{Variables fisiológicas}

$\mathrm{Al}$ relacionar la asincronía floral con el rendimiento de biomasa total por planta (Figura 3), se observó que conforme disminuyó la asincronía, el rendimiento de biomasa se abatió considerablemente, manteniéndose más alto en las variedades mejoradas. Sobre este particular, NeSmith y Ritchie (1992), encontraron en plantas de maíz, una reducción de la biomasa y rendimiento entre 21 y $40 \%$ por efecto de la sequía, siendo el peso del grano la variable más afectada, ya que se acortó en ocho días el periodo de llenado del mismo, debido, según Westgate (1994), a un secado prematuro del endospermo y a un volumen limitado del embrión. Por otra parte, Ouattar et al. (1987a), así como Cheikh y Jones (1994), mencionan que el crecimiento del grano es muy sensible al déficit hídrico, especialmente en la primera fase, donde se da la división celular del endospermo. Lo anterior se encuentra estrechamente relacionado con el índice de cosecha (IC), el cual se redujo hasta en 2,4 veces para Co, 3,3 veces para C16, 1,6 para Z0 y 3,0 para Z19 respecto del tratamiento de riego.

La diferencia entre variedades dentro de cada tratamiento fue muy clara, ya que las variedades originales presentaron mayor índice de cosecha que las mejoradas (Cuadro 2). Esta disminución del IC en las variedades mejoradas pudo deberse a que éstas durante la sequía distribuyeron más biomasa a la raíz que las originales para aumentar la exploración de la humedad del suelo. Es así como se explica que la relación raíz-vástago de las variedades mejoradas fue mayor que las de las variedades originales (Cuadro 3); además, el índice de sequía se mantuvo por arriba de la unidad, indicando con ello un cierto grado de resistencia a la sequía (Cuadro 2 y 3). Al respecto Fischer y Turner (1978), 


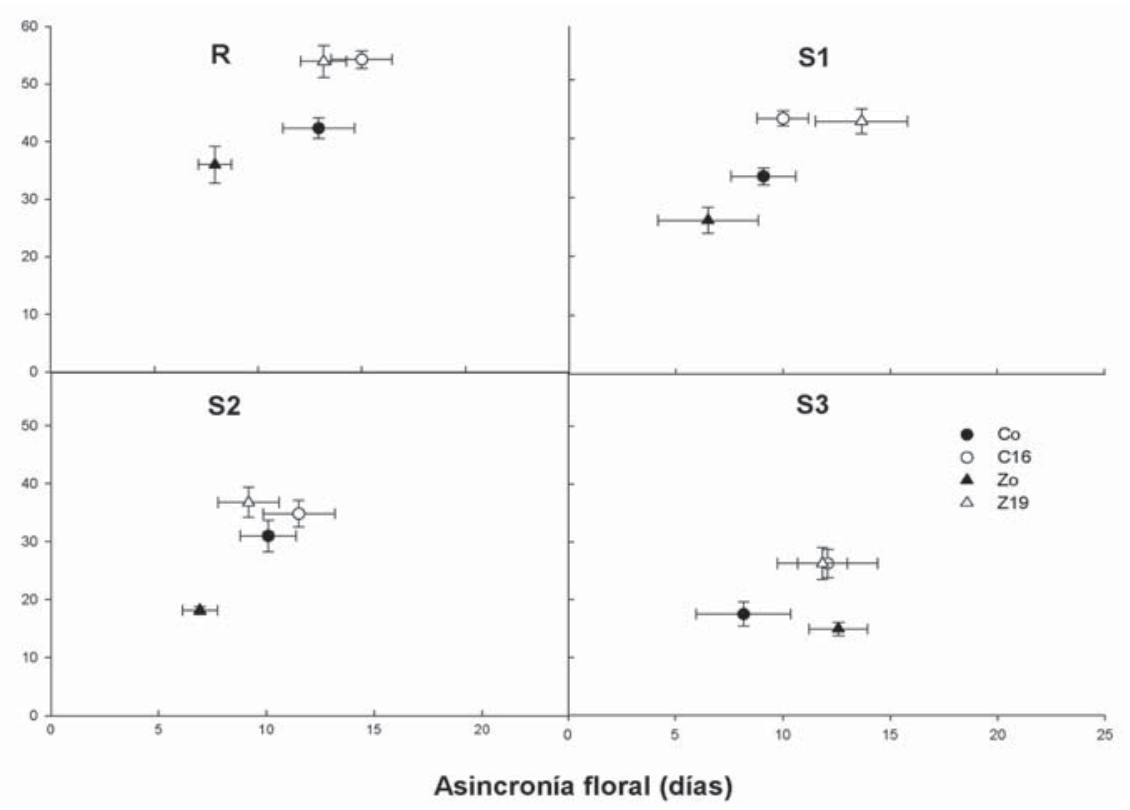

Figura 3. Relación entre la biomasa total y la asincronía en cuatro variedades de maíz, bajo riego (R) y tres niveles de sequía (S1, S2, S3) a 4, 10 y 20 días después de la condición de punto de marchitez permanente (PMP), respectivamente. Cada punto es el promedio de tres repeticiones \pm error estándar. Colegio de Postgraduados, México. 2003.

mencionan que bajo sequía, ocurre un mayor aumento de la materia seca en la raíz que en el vástago, lo que permite una exploración radical extra, reduciéndose en consecuencia el índice de cosecha. Sin embargo, estos autores también mencionan que la exploración radical y el índice de cosecha pueden ser antagónicos.

\section{CONCLUSIONES}

Las variedades mejoradas obtenidas por selección masal en condiciones de baja humedad edáfica para resistencia a sequía evaluadas en el presente trabajo, mostraron mayor capacidad de recuperación que las no mejoradas una vez que cesó el estrés hídrico. La sequía retrasó las floraciones masculina y femenina; sin embargo, la asincronía floral fue mayor en las variedades mejoradas. El índice de cosecha de las variedades mejoradas fue menor que el de las no mejoradas, como consecuencia de una mayor acumulación de biomasa en la raíz que en la parte aérea. El detenimiento del crecimiento vegetativo por efecto del estrés hídrico en las variedades mejoradas y su rápida recuperación, una vez cesado el estrés, fue un indicador de que dichas variedades durante el proceso de selección, han desarrollado mecanismos de resistencia que les permiten sobrevivir en condiciones de baja humedad.

\section{LITERATURA CITADA}

Avendaño, ACH; Trejo, LC; López, CC; Molina, GJD; Santacruz, VA; Castillo, GF. 2005. Comparación de la tolerancia a la sequía de cuatro variedades de maíz (Zea mays L.) y su relación con la acumulación de prolina. Interciencia 30(9): 560-564.

Bassetti, P; Westgate, ME. 1993. Water deficit affects receptivity of maize silks. Crop Science 33: 279-282.

Boyer, JS; Westgate, ME. 2004. Grain yields with limited water. Journal of Experimental Botany 55(407): 2385 2394.

AGRONOMÍA MESOAMERICANA 19(1): 27-37. 2008 
Bradford, KJ. 1994. Water stress and the water relations of seed development: A critical review. Crop Science 34: 1-11.

Cheikh, N; Jones, R. 1994. Disruption of maize kernel growth and development by heat stress. Plant Physiology 106: 45.51 .

Claassen, MM; Shaw, RH. 1970. Water deficit effects on corn. II. Grain components. Agronomy Journal 62: 652-655.

Desai, SA; Singh, RD. 2001. Combining ability studies for some morphophysiological and biochemical traits related to drought tolerance in maize (Zea mays L.). Indian Journal Genetic Plant Breeding 61: 34-36.

Ekanayake, IJ; Steponkus, PL; De Datta, SK. 1990. Sensitivity of pollination to water deficits at anthesis in upland rice. Crop Science 30: 310-315.

Fischer, KS; Turner, NC. 1978. Plant productivity in the arid and semiarid zones. Annual Review Plant Physiology 29: $277-317$.

García, E. 1988. Modificaciones al sistema de clasificación climática de Koppen (Para adaptarlo a las condiciones de la República Mexicana). Ed. Instituto de Geografía. UNAM, México, D.F. 217 p.

Graff, DF. 1989. Responses of desiccation tolerant "resurrection" plants to water stress. In: Structural and functional responses to environmental stresses. K H Krebb, H. Richter, T M Hinkley. eds.SPB Academic Publishers, The Hague, The Netherlands. p. 255-268.

Herrero, MP; Jonhson, RR. 1981. Drought stress and its effects on maize reproductive systems. Crop Science 21: $105-110$.

Molina, GJD. 1980. Selección masal para resistencia a sequía en maíz. Agrociencia 42: 69-77.

Moss, GI; Downey, LA. 1971. Influence of drought stress on female gametophyte development in corn (Zea mays L.) and subsequent grain yield. Crop Science 11: 368-372.
Muñoz, OA; Stevenson, KR; Ortiz, CJ; Thurtell, GW; Carballo, CA. 1983. Transpiración, fotosíntesis, eficiencia en uso de agua y potencial hídrico en maíces resistentes a sequía y a heladas. Agrociencia 51: 11-153.

Namuco, OS; O'toole, JC. 1986. Reproductive stage water stress and sterility. I. Effect of stress during meiosis. Crop Science 26: 317-321.

Neale, AD; Blomstedt, CK; Bronson, P; Le, TN; Guthridge, K; Evans, J; Gaff, DF; Hamill, JD. 2000. The isolation of genes from the resurrection grass Sporobolus stapfianus wich are induced during severe drought stress. Plant Cell Enviroment 23: 265-277.

NeSmith, DS; Ritchie, JT. 1992. Maize (Zea mays L.) response to a severe soil water-deficit during grain-filling. Field Crops Research 29: 23-35.

O'Toole, JC; Namuco, OS. 1983. Role of panicle exsertion in water stress induced sterility. Crop Science 23: 1093-1097.

O'Toole, JC; Moya, TB. 1981. Water deficits and yield in upland rice. Field Crops Research 4: 247-259.

Ober, ES; Setter, TL; Madison, JT; Thompson, JF; Shapiro P. 1991. Influence of water deficit on maize endosperm development. 97: 154-164.

Ouattar, SR; Jones, J; Crookston, RK. 1987a Effect of water deficit during grain filling on the pattern of maize kernel growth and development. Crop Science 27: 726-730.

Outtar, SR; Jones, J; Crookston, RK; Kajeiou, M. 1987 b. Effect of drought on water relations of developing maize kernels. Crop Science 27: 730-735.

Saini, SH; Westgate, ME. 2000. Reproductive development in grain crops during drought. Advances in Agronomy 68: 59-96.

Sawhney, VK; Shukla, A. 1994. Male sterility in flowering plants: are plant growth substances involved?. American Journal of Botany 81(12): 1640-1647. 
Schoper, JB; Lambert, RJ; Vasilas, BL. 1986. Maize pollen viability and ear receptivity under water and high temperature stress. Crop Science 26: 1029-1033.

Schussler, JR Westgate, ME. 1991 Maize kernel set at low water potential: I. Sensitivity to reduced assimilates during early kernel growth. Crop Science 31: 11891195.

Schussler, JR; Westgate, ME. 1995. Assimilate flux determines kernel set at low water potential in maize. Crop Science 35: 1074-1080.
Setter, TL; Flannigan, BA; Melkonian, J. 2001. Loss of kernel set due to water deficit and shade in maize: carbohydrate supplies, abscisic acid, and cytokinins. Crop Science 41: 1530-1540.

Westgate, ME. 1994. Water status and development of the maize endosperm and embryo during drought. Crop Science 34: 76-83.

Zinselmeier, C; Lauer, MJ; Boyer, JS. 1995. Reserving droughtinduced losses in grain yield: Sucrose maintains embryo growth in maize. Crop Science 35: 1390-1400. 